\title{
Correlative t-EBSD Tomography and Atom Probe Tomography Analysis
}

\author{
Y. Chen ${ }^{1}$, K.P. Rice ${ }^{1}$, T.J. Prosa ${ }^{1}$, M.M. Nowell ${ }^{2}$ and S.I. Wright ${ }^{2}$ \\ 1. CAMECA Instruments, Inc., Madison, WI, USA \\ 2. EDAX, Draper, UT, USA
}

Atom probe analysis (APT) has the capability of providing compositional analysis at the atomic-scale but can suffer from inaccuracies in the 3D reconstruction. This is generally due to the fact that the tip geometry and the electric field distribution at tip apex are almost impossible to measure accurately in real time. As a result, APT reconstructions are usually based on a number of assumptions; e.g., assuming a hemi-spherical apex, and/or a single point projection center, with the field estimate based on either experimental or theoretical results. In practice, features with well-known geometries that are present in the reconstructed volume are routinely used to help improve reconstruction accuracy. The most common features are phase boundaries, grain boundaries, and spherical precipitates. In order to observe and measure these features at the necessary spatial resolution, high resolution SEM or TEM is routinely used to confirm feature geometry. Preferably, such techniques are used directly on the tip specimens that are then analyzed by APT. APT analysis has a limited analyzable volume which is typically a few hundred nanometers from the apex depending on the tip geometry and the material type. Ensuring a feature of interest can be captured within that volume is essential to APT analysis.

Efforts in correlating atom probe analysis with other analytical techniques have been made for targeting, improved reconstruction accuracy, and to obtain crystallographic information. Arslan et al. [1] has successfully demonstrated STEM tomography in conjunction with APT analysis on a single tip specimen. This type of comparison allows direct visualization of the theoretically known artifacts of each technique experimentally, and provides insight to the optimal parameters for reconstructions. Similarly, the usage of nanobeam diffraction (NBD) in STEM has been reported to measure grain orientations, while subsequent APT analysis is used to quantitatively measure grain boundary chemistry. Combining these techniques provide a robust grain boundary chemistry analysis on crystalline materials with adequate grain sizes and allows for correlation between grain boundary misorientation and chemical segregation.

In this work we introduce a new type of correlative analysis by joining transmission EBSD (tEBSD/TKD) tomography and APT. Transmission-EBSD analysis is uniquely suited to needle-shaped APT specimens with regard to the sample thickness requirement for electrons to scatter and penetrate [2]. Previous studies have demonstrated the two techniques are complementary in terms of acquiring crystallographic information using EBSD and atomic-scale composition information using APT [3]. In comparison to the aforementioned correlative analyses with STEM, t-EBSD analysis can be rapidly performed between FIB millings without the need to break vacuum. Transmission-EBSD mapping can also provide real time feedback to guide the FIB milling process, so particular regions of interest for APT analysis can be targeted quickly and accurately.

Transmission EBSD patterns are known to be generated from the near surface where electrons exit the specimen. Transmission-EBSD-tomography analysis requires the ability to rotate specimen along the tip axis and map the specimen sequentially. Specimens can be mounted on typical half-grid or wire holders. The chamber stage or an additional mountable rotation arm such as a Kleindiek can both be used for the 
tomographic rotation. Figure 1 shows sequential t-EBSD maps of the same APT specimen with a series of rotation angles. The change in color represents crystallographic orientation rotation along the tip axis. This tip contains two grains with a significant size difference. The smaller grain near the apex is not always visible at all the rotations. At the rotation angle of $270^{\circ}$ from the initial mapping position ,only the larger grain can be observed due to the small grain being rotated to the top side, so the Kikuchi diffraction patterns observed on the detector are overwhelmed by the large grain which occupies the exit surface of the tip. Looking at rotations between 0 and $135^{\circ}$, one can see the small grain gradually decreasing in size as it moves to the top surface. The small grain shows poorer image quality (IQ) due to the pattern overlap issue, and in many rotation angles the small grain didn't get indexed correctly due to pattern overlap.

Taking t-EBSD maps at various rotation angles and stitching them together allows the grain configuration to be visualized in 3D. Transmission-EBSD tomography and atom probe tomography are complementary because t-EBSD only detects orientation information from grains at the near surface region, and APT gives the compositional information in the core region of the tip volume. The correlative analysis of the two techniques will provide a new level of microstructure analysis to obtain both crystallographic information and local chemistry information at the nanoscale.

\section{References:}

[1] I. Arslan, et al, Ultramicroscopy 108.12 (2008) p. 1579.

[2] K.P. Rice, R.R. Keller, M.P. Stoykovich, Journal of Microscopy 254 (2014) p. 129.

[3] K. Babinsky et al, Ultramicroscopy 159(2) (2015) p. 445.

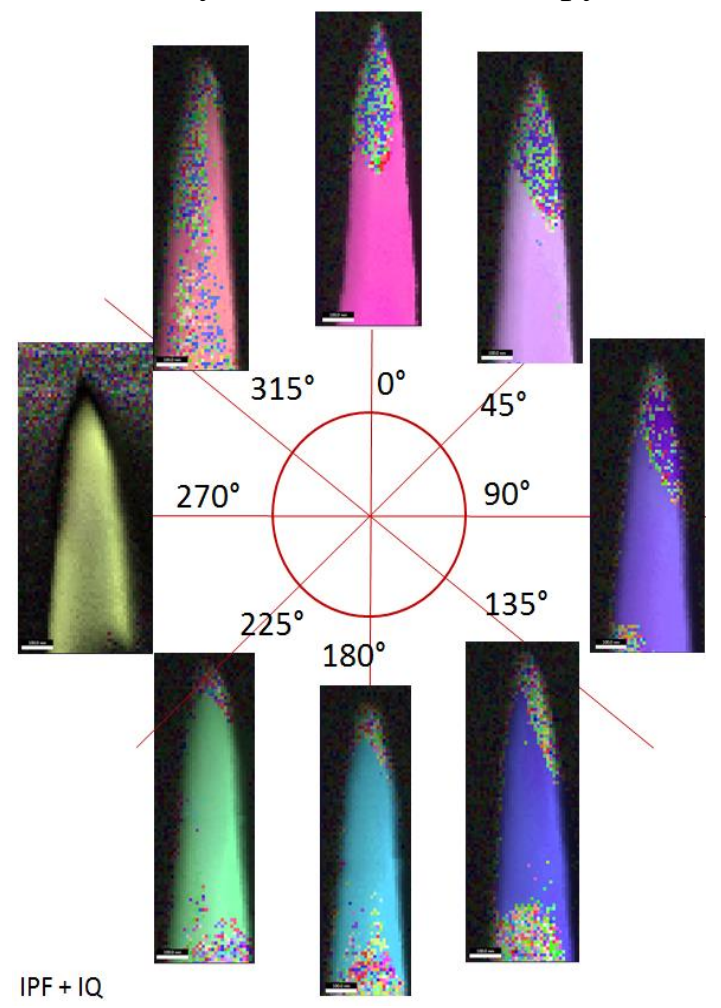

Figure 1. t-EBSD normal direction inverse pole figure maps with image quality overlay of Inconel 600. Angles indicated degree of rotation along the tip axis from the initial position.

\section{T-EBSD map APT map}

(a)

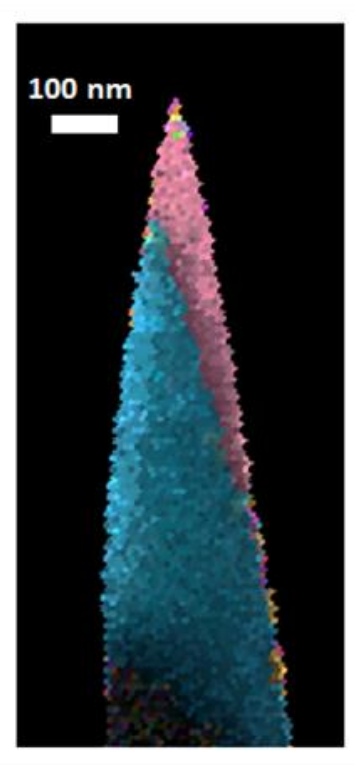

(b)

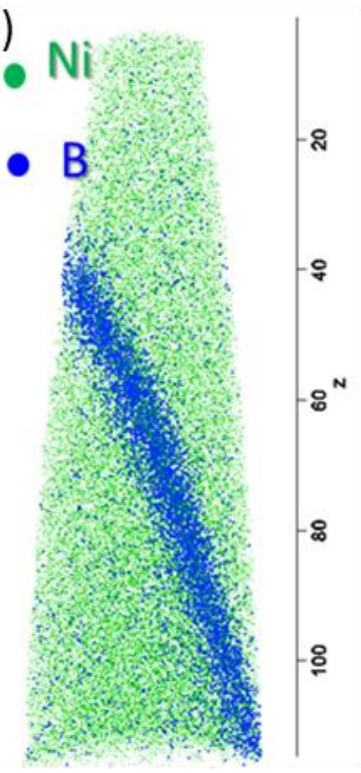

Figure 2. (a) t-EBSD inverse pole figure and image quality map of Inconel 600; (b) APT atom maps showing boron segregation in the grain boundary. 